\title{
The controversial origin of pericytes during angiogenesis - Implications for cell-based therapeutic angiogenesis and cell-based therapies
}

\author{
Anna Blocki ${ }^{\mathrm{a}, \mathrm{b}, *}$, Sebastian Beyer ${ }^{\mathrm{a}}$, Friedrich Jung ${ }^{\mathrm{c}}$ and Michael Raghunath ${ }^{\mathrm{d}, *}$ \\ ${ }^{\mathrm{a}}$ Institute for Tissue Engineering and Regenerative Medicine, Chinese University of Hong Kong, \\ Hong Kong SAR \\ ${ }^{\mathrm{b}}$ School of Biomedical Science, Faculty of Medicine, Chinese University of Hong Kong, \\ Hong Kong SAR \\ ${ }^{\mathrm{c}}$ Institute for Clinical Hemostasiology and Transfusion Medicine, University Saarland, \\ Homburg/Saar, Germany \\ ${ }^{\mathrm{d}}$ Institute of Chemistry and Biotechnology, Zurich University of Applied Sciences, \\ Wädenswil, Switzerland
}

\begin{abstract}
Pericytes reside within the basement membrane of small vessels and are often in direct cellular contact with endothelial cells, fulfilling important functions during blood vessel formation and homeostasis. Recently, these pericytes have been also identified as mesenchymal stem cells. Mesenchymal stem cells, and especially their specialized subpopulation of pericytes, represent promising candidates for therapeutic angiogenesis applications, and have already been widely applied in pre-clinical and clinical trials. However, cell-based therapies of ischemic diseases (especially of myocardial infarction) have not resulted in significant long-term improvement. Interestingly, pericytes from a hematopoietic origin were observed in embryonic skin and a pericyte sub-population expressing leukocyte and monocyte markers was described during adult angiogenesis in vivo. Since mesenchymal stem cells do not express hematopoietic markers, the latter cell type might represent an alternative pericyte population relevant to angiogenesis. Therefore, we sourced blood-derived angiogenic cells (BDACs) from monocytes that closely resembled hematopoietic pericytes, which had only been observed in vivo thus far. BDACs displayed many pericytic features and exhibited enhanced revascularization and functional tissue regeneration in a preclinical model of critical limb ischemia. Comparison between BDACs and mesenchymal pericytes indicated that BDACs (while resembling hematopoietic pericytes) enhanced early stages of angiogenesis, such as endothelial cell sprouting. In contrast, mesenchymal pericytes were responsible for blood vessel maturation and homeostasis, while reducing endothelial sprouting.

Since the formation of new blood vessels is crucial during therapeutic angiogenesis or during integration of implants into the host tissue, hematopoietic pericytes (and therefore BDACs) might offer an advantageous addition or even an alternative for cell-based therapies.
\end{abstract}

\section{Introduction}

Small blood vessels are composed of an inner lining formed by endothelial cells and pericytes that share their basement membrane. These two cell-types are often found in direct cell-cell contact [1,2]. Pericytes were discovered as early as 1873 [3], nevertheless they still represent an elusive cell-type.

\footnotetext{
${ }^{*}$ Corresponding authors: Anna Blocki, Institute for Tissue Engineering and Regenerative Medicine \& School of Biomedical Science, Faculty of Medicine, Chinese University of Hong Kong, Hong Kong. E-mail: anna.blocki@cuhk.edu.hk and Michael Raghunath, Institute of Chemistry and Biotechnology, Zurich University of Applied Sciences, Wädenswil, Switzerland. E-mail: ragh@zhaw.ch.
} 
Pericytes are indispensable from normal blood vessel development and function. They contribute to vessel remodelling, architecture, permeability and regulate blood flow [4]. During angiogenesis pericytes are recruited from the bone marrow as well as surrounding tissue [5-8] via PDGFR- $\beta$ signalling $[9,10]$ and are crucial for vessel formation, maturation and proper function $[11,12]$. Nonetheless, their exact role during angiogenesis still remains obscure. Knock-down models of PDGF-B and PDGFR- $\beta$ demonstrated that the absence of pericytes did not affect angiogenesis [13]. In addition, pericytes negatively regulate endothelial cell proliferation, which represents an essential stage during vessel sprouting $[14,15]$. Currently it is believed that pericytes are needed to detach from vessels to initiate endothelial sprouting [16].

In contrast to these observations, inhibition of pericyte recruitment into tumours was associated with increased endothelial cell apoptosis and a reduction in tumour growth [5, 17]. Similarly, inhibition of pericyte recruitment in a skin wound healing model resulted in a reduction of vessel ingrowth [18]. In addition, pericytes have been observed to modulate sprouting endothelial cells and anastomosis $[19,20]$.

Currently there are no pericyte-specific markers available and pericytes exhibit a heterogeneous expression of the commonly applied markers [21]. In addition to their heterogeneity in marker expression, they have different embryonic origins (including mesodermal, neuroectodermal and endothelial) [4] and participate in highly specialized functions dependent on their location [21, 22].

Although pericytes are mainly identified by their perivascular location and not by their phenotype, have a diverse marker expression profile and seem to fulfil various functions, it is currently assumed that pericytes represent a heterogeneous population of cells [21]. Pericyte subpopulations with specialized functions can be found in a number of different tissues, which is dependent on their temporal and spatial distribution $[21,23]$.

Pericytes have been identified to resemble mesenchymal stem cells (MSCs) and the perivascular location was proposed to act as a universal stem cells niche [24, 25]. However, several independent groups have reported that during early stages of angiogenesis and vessel development, pericytes have a hematopoietic phenotype [5, 6, 26-30]. It is important to note that MSCs by definition do not express hematopoietic markers [31]. Thus, it may be hypothesized that pericyte populations have distinct origins with specialized functions during angiogenesis.

\section{Pericytes - recruitment and function}

\subsection{During embryogenesis}

A large body of our current understanding about pericyte recruitment and function is derived from studies during embryonic development. Knock-out models of PDGF-B and PDGFR- $\beta$ genes showed a lack of PDGFR- $\beta$ expressing pericytes around microvessels, whereas coverage of larger vessels by smooth muscle cells was not affected $[32,33]$. Both knock-out models demonstrated almost identical outcomes characterized by dilated, non-functional and leaky blood vessels resulting in haemorrhages and oedema, leading to premature perinatal death $[9,10]$. Microvessels in these knock-out models as well as additional knock-down models of PDGF-B and PDGFR- $\beta$ exhibited an abnormal morphology with endothelial processes protruding into the vessel lumen and variable thickness of endothelium. The mean vessel diameter was increased and it appeared that small blood vessels contained an increased number of endothelial cells [13]. Thus, pericytes were implicated to negatively control endothelial cell proliferation, induce endothelial cell maturation and regulate microvessel integrity, structure and thereby proper function during development $[11,13,32]$. 
Interestingly, knock-down of PDGF-B and PDGFR- $\beta$ suggested that pericytes did not affect all stages of angiogenesis such as capillary sprouting, as the number of capillaries, their branching points and also microvessel length appeared comparable to controls [13]. In vitro experiments provided further evidence on the inhibitory effects of pericytes on endothelial cell proliferation. This effect appeared to be pericyte-specific; in contrast, other mesenchymal cells such as fibroblasts enhance endothelial cell proliferation [14].

\subsection{During adulthood}

During adulthood angiogenesis occurs mainly in tumours and during wound healing. Interestingly, vasculature in various tumours and even more so after inhibition of pericyte recruitment, exhibited a phenotype that was comparable to that of PDGF-B or PDGFR- $\beta$ deficient mice [17]. Small blood vessels had variable, and mostly larger diameters, with increased permeability. The irregularity of blood vessels in tumours again correlated with a decrease in the coverage of blood vessels by loosely attached pericytes $[5,17]$. The absence of pericytes led to an increase of apoptotic cells in the tumour, most of them being endothelial cells [5].

Inhibition of pericyte recruitment, in a skin wound model, resulted in a reduction of pericyte proliferation and in an overall decrease in pericyte number. In addition less infiltrating blood vessels were observed in these wounds and formed vessels appeared dilated [34].

Thus, it can be concluded that pericytes are indispensable for blood vessel formation, maintenance and function during embryonic development and adulthood. Furthermore, our knowledge about pericyte function during embryonic development allows to a certain extent to predict their effect on blood vessel formation and homeostasis during adulthood.

\subsection{Signalling pathways implicated in pericyte recruitment and function}

Several excellent reviews have elaborated the various pathways involved in the development of pericytes and their interaction with endothelial cells $[12,35]$. Thus, the most prominent features will be summarized in the following sections.

\subsubsection{PDGF-B/PDGFR- $\beta$ signalling}

Sprouting endothelial cells secrete PDGF-B in form of a homodimer that binds to heparin sulphate proteoglycans on the surface of endothelial cells and incorporated into the extracellular matrix. PDGF$B$ recruits pericytes that in turn express PDGFR- $\beta$ on their cell surface $[12,35]$. Recently, CD146 was recognized as a co-receptor of PDGFR- $\beta$ and its importance for pericyte recruitment was verified [36].

\subsection{2. $T G F-\beta$ signalling}

Activin receptor-like kinases (ALK)-1 and 5 are TGF- $\beta$ receptors and expressed on endothelial cells and pericytes. These receptors are currently assumed to have opposing effects. Alk-1 promotes proliferation and migration of mesenchymal cells, whereas Alk-5 is known to inhibit proliferation and migration and induce differentiation. Moreover, the function of TGF- $\beta$ is complex and the cellular response strongly depends on the interplay between endothelial cells and pericytes and resulting secondary signalling responses, as well as the interaction between the signalling pathways activated through Alk-1 and Alk-5. Currently it is suggested that both Alk receptors become activated during moderate TGF- $\beta$ signalling. During the sprouting phase Alk-1 inhibits the Alk-5 pathway and induces the expression of genes responsible for cell migration, proliferation and tube 
formation. After prolonged TGF-ß stimulation Alk-1 signalling decreases and Alk- 5 becomes predominant, inducing vessel maturation including cell differentiation and basement membrane production $[12,35]$.

\subsubsection{Angiopoietin/Tie-2 signalling}

Angiopoietin (Ang)-1 represents the agonist and Ang-2 the antagonist for the receptor Tie-2, which is expressed on the surface of endothelial cells. Ang-1 is mainly secreted by perivascular cells such as pericytes and induces endothelial cell survival and vessel stabilization, whereas Ang-2 that is mainly secreted by the endothelium itself in areas of vascular remodelling and has opposing effects. Ang-1 is believed to induce endothelial cell stability and quiescence, while Ang-2 induces pericyte detachment during angiogenesis $[12,35]$.

Very recently the role of pericyte-expressed Tie-2 was accentuated. Pericyte-specific silencing of Tie2 as well as stimulation by the antagonist Ang- 2 significantly increased pericyte motility. Additionally, Tie-2-silenced pericytes did not inhibit endothelial sprouting in an in vitro and in vivo model, when compared to their Tie-2-active opponents. Pericyte-specific Tie-2 deletion transiently delayed retinal vascularization during development and reduced the amount of pericytes covering blood vessels. When subcutaneous tumours were introduced into this mouse model, vessels appeared more angiogenic and leaky as compared to controls [16]. Hence pericyte-expressed Tie-2 seemed also to contribute to vessel formation, maturation and homeostasis.

\subsection{Pericytes - a population of mesenchymal stem cells (MSCs)?}

Generally, pericytes are identified by their specific location in vivo, whereas MSCs are identified in vitro. The minimal criteria for identification of MSCs as defined by the scientific community for cellular therapy is the ability of the cell to adhere to plastic, to express CD105, CD73, CD90 and lack the expression hematopoietic markers CD45, CD34, CD14 or CD11b, CD79 $\alpha$ or CD19, HLA-DR, as well as differentiate into the three mesenchymal lineages osteoblasts, adipocytes and chondrocytes under standard in vitro conditions [31].

Pericytes have long been suspected to act as mesenchymal progenitors. In 1990's it was shown that pericytes from bovine retina grown to confluency formed multi-layered areas, which differentiated into multicellular nodules containing collagen fibres and hydroxyapatite, indicating osteoblasts [37]. Pericytes from bovine brain microvasculature were induced using a standard osteogenic protocols for MSCs and formed colonies that synthesised alkaline phosphatase, hydroxyapatite, collagen, glycosaminoglycans and most importantly osteocalcin, identifying them as osteoblasts [38, 39].

Retinal pericytes were also differentiated into chondrocytes using a standard chondrogenic protocol for MSCs, and into adipocytes using rabbit serum in vitro. In vivo transplantation using diffusion chambers demonstrated that their chondrogenic and adipogenic differentiation potential was not restricted to in vitro conditions [40].

Crisan et al. were the first group to systematically analyse MSC features in pericytes derived from various tissues [41]. They identified CD146 expressing cells in the perivascular space that were distinguishable from endothelial cells by the lack of CD34 expression, as pericytes. These cells could be isolated from a variety of tissues (e.g. skeletal muscle, myocardium, placenta, pancreas, skin, brain, bone marrow and white adipose tissue) and made up $0.88 \%$ (muscle) to $14.6 \%$ (adipose) of total cells harvested from respective tissues. They expressed MSC markers immediately after isolation and longterm expansion. Most importantly, these cells were able to differentiate into adipocytes, osteoblast and chondrocytes under standard MSC induction conditions, even at a clonal level [41]. A small fraction of cardiac pericytes was even demonstrated to be able to differentiate into cardiomyocyte in vitro and in vivo by the same group [42]. Therefore, isolated pericytes were able to fulfil the criteria, which were 
assigned as MSCs. Thus, it was hypothesised that the perivascular location holds a reservoir of MSCs that are recruited during wound repair and tissue regeneration [25].

\subsection{Regenerative effects of pericytes}

The regenerative potential of endogenous pericytes was studied as early as 1992, when pericytes and endothelial cells were exclusively labelled in vivo with Monastral blue [43]. Without damaging the surrounding microvasculature, the periosteum strip was lifted in an adult rat femur and thus bone formation was induced. Shortly thereafter, some of the previously labelled pericytes were detected in the newly formed bone resembling osteoblasts [43]. By utilizing genetic lineage tracing Feng and colleagues showed that pericytes can differentiate into odontoblasts (specialized mesenchymederived cells) during tooth repair [44]. Moreover, it was discovered that a subset of pericytes, identified by the established markers $\alpha$-SMA, PDGFR- $\beta$ and NG2, are a source of adipocytes during murine development [45]. When delivered exogenously, transplanted pericytes gave rise to numerous muscular fibres in mice with muscular dystrophy or after muscle injury with cardiotoxin [41, 46, 47], and enhanced early wound healing in a full thickness skin wound [48]. In addition, pericytes were long implicated to play a role in wound healing as well as fibrosis by differentiating into myofibroblasts [49]. The current results strongly suggest that at least a subset of pericytes have regenerative potential and differentiates into other lineages to restore or replenish tissue.

This concept was recently opposed by Guimares-Camboa et al. They claimed that Tbx18, in contrast to previously utilized pericyte lineage tracking markers in other studies, was truly pericyte-specific. Using Tbx18 as their chosen marker for genetic lineage tracing they demonstrated that pericytes did not significantly contribute to other cell lineages such as adipogenic, fibrotic or myogenic progenitors but remained pericytes throughout aging and tissue repair in vivo although they were multipotent in vitro. They thus concluded that pericytes did not behaved as innate MSCs in vivo [50, 51]. Further they claimed that Tbx18 was labelling all PDGFR-beta perivascular cells in heart, brain, skeletal muscle and adipose tissue. Interestingly, this marker did not label any cells in highly vascularized and pericytecarrying tissues such as kidney, liver, small and large intestines [50]. As pericytes are known to be extremely heterogeneous with even different embryonic origins [4, 29, 30], the question of Tbx18 labelling all pericytes remains to be verified. Birbair et al, for example, described two subpopulations of pericytes in the brain, distinguishable by their expression of nestin. They found that only pericytes lacking nestin contributed to scar tissue formation after brain injury [52].

\section{Are all MSCs pericytes?}

Since pericytes fulfil specialized functions differing from activities associated with differentiation into other mesenchymal lineages, Caplan (2008) suggested that not all pericytes are MSCs; however, he implied that all MSCs are pericytes and raised the question, whether pericytes would contribute to tissue repair by differentiating into other lineages in vivo [53].

Indeed, it was confirmed that similar to pericytes, MSCs can be isolated from various tissues and a further suggestion of a systemic reservoir of MSCs in the perivascular space was formulated [54].

Since MSCs are known to be a heterogeneous cell population [55] the question remains if indeed all MSCs can act as pericytes. This question was recently addressed in a review and concluded that there are other perivascular cells, which do not resemble pericytes, but can act as MSCs [56]. Shortly thereafter the authors published their research findings, in which they described the isolation of CD34 positive cells from the tunica adventitia of arteries and veins from adipose tissue, which could be distinguished from endothelial cells, leukocytes and pericytes, as they lacked the expression of CD31, 
CD45 and CD146, respectively [57]. Isolated cells expressed MSC markers CD44, CD73, CD105 and CD90 in vitro as well as in vivo and differentiated into adipocytes, osteoblasts and chondrocytes. Therefore, it was established that adventitial cells, although MSC in nature, are anatomically and phenotypically distinct from pericytes. However, when treated with AugTP2, they could be induced to express pericyte markers PDGFR- $\beta$, CD146, $\alpha$-SMA and NG2 [57]. Feng et al. (2011) further demonstrated that pericytes, which gave rise to odontoblasts, were accompanied by MSCs from a non-pericytic origin doing the same [44]. Nonetheless, the question if all MSCs can act as pericytes remained unclear, which was subsequently addressed by some of us. Pericytes $\left(\mathrm{CD} 146^{+} \mathrm{CD}^{-} 4^{-}\right)$and all associated non-pericytic MSCs $\left(\mathrm{CD} 146^{-} \mathrm{CD}^{-}\right)$were isolated from human bone marrow, treated identically during cell expansion as well as during all assays and were compared in terms of marker expression, differentiation capacity and functional behaviour in various angiogenesis assays [58]. In our hands, pericytes proliferated slower, when compared to non-pericytic MSCs from the same bone marrow samples. However, we were not able to distinguish pericytes and non-pericytic MSCs in terms of marker expression and differentiation capacity after in vitro cultivation. Interestingly, non-pericytic MSCs gained the expression of CD146, although they were initially selected due to absence of this marker [58]. Moreover when these cell-types were subjected to functional angiogenesis assay, it became evident that only pericytes were able to stabilize endothelial tubular networks and sprouting, whereas non-pericytic MSCs had no effects when cultivated in direct contact with endothelial cells [58]. Thus, it may be concluded that from the functional point of view, not all MSCs can act as pericytes.

\section{Are all pericytes MSCs?}

Pericytes fulfil many specialized functions not associated with differentiation into other lineages as it is expected from MSCs [22]. In addition, they exhibit a divergent marker expression profile dependent on their temporal and spatial distribution [21]. Currently, there is active discussion in the scientific community whether all pericytes can act as MSCs. Interestingly, it was already demonstrated that pericyte populations with various capacities to differentiate exist. Two pericyte subpopulations that were distinguished by their differential expression of nestin, gave rise exclusively to either myoblasts or adipocytes following muscle injury [59]. Thus, heterogeneity even exists in MSC-like pericytes.

Furthermore, several independent groups have reported pericytes and other related cell types such as smooth muscle cells to have hematopoietic features, which MSCs by definition do not possess [31].

Just recently Yamazaki and colleagues (2017) performed genetic fate mapping experiments to determine the origin of pericytes in embryonic skin and brain during development. They found that neural crest and endothelial cells did not significantly give rise to pericytes, whereas a subpopulation of $\mathrm{NG}^{+}$pericytes (accounting for 27\%) was derived from Vav-expressing thus hematopoietic cells. Although these pericytes did not express CD11b or F4/80 anymore, around $13 \%$ of all pericytes were further demonstrated to be derived from CD11b expressing cells. Isolated dermal myeloid progenitors even differentiated into pericytes in vitro and TGF $\beta$ signalling was implicated to play a significant role in this differentiation process. Interestingly the PU.1 $1^{-/}$mouse strain, which basically lacks macrophages, also exhibited a drastic reduction of pericytes around blood vessels, indicating that cells from hematopoietic origin contribute to pericyte function in ectodermal organs. The researchers concluded that distinct pericyte populations with distinct origins and thus potential roles and functions exist [30].

Noteworthy, pericytes with a hematopoietic phenotype were also observed during adulthood in various situations of angiogenesis; During neo-intimal hyperplasia resulting from smooth muscle cell proliferation at the site of luminal vessel injury, these proliferating cells expressed hematopoietic mark- 
ers [60]. When lethally irradiated mice with localised vessel injuries received purified hematopoietic stem cells (HSCs), it was demonstrated that the neointimal hyperplasia was fuelled by cells that were indeed newly derived from HSCs that repopulated the bone marrow upon transplantation [61].

During embryogenesis, HSCs that appear in the brain ectodermal layer were reported to guide and stabilize blood vessels. Upon isolation for further in vitro culture, a small fraction of these cells differentiated into cells expressing pericyte markers, PDGFR- $\beta$ and $\alpha$-SMA [62]. However, this phenomenon was not restricted to embryogenesis. During adulthood, when peripheral blood cells expressing leukocyte marker CD45 and monocyte marker Cd11b were derived from animals with critical hind limb ischemia, they differentiated into $\alpha$-SMA expressing cells in vitro. In vivo these cells were associated with blood vessels and expressed pericyte markers, PDGFR- $\beta$ and $\alpha$-SMA [62].

During angiogenesis induced by VEGF injection into ears of mice that had their bone marrow reconstituted with $\mathrm{GFP}^{+}$cells, the majority of peri-endothelial cells were shown to originate from the bone marrow and express pericyte marker NG2, pan-leukocyte marker CD45 and monocyte/macrophage marker CD11b [6]. Similarly, when angiogenesis was induced by injection of bFGF into the cornea, majority of pericytes around forming vessels identified by PDGFR- $\beta$ and NG2 were also derived from the bone marrow and co-expressed CD45 and CD11b [26]. Song et al. [5] found that a proportion of PDGFR- $\beta$ expressing pericyte progenitors co-expressed CD11b also during tumour angiogenesis [5]. Subsequently it was proposed in a review by Lamagna and colleagues that pericytes were not only derived from the bone marrow, but also had a haematopoietic origin [63]. Tigges and colleagues monitored infiltrating cells at various stages of angiogenesis using an in vivo matrigel plug supplemented with bFGF [28]. Interestingly, the initial cellular network was free of CD31 expressing endothelial cells and was not perfused. The majority of infiltrated cells expressed NG2 and were therefore identified as pericytes. A high proportion of these cells were bone marrow-derived and co-expressed CD45, and a quarter of these cells also expressed the macrophage marker F4/80. Although, macrophages $\left(\mathrm{F} 4 / 80^{+}\right)$ that did not express NG2, persisted in the plug, they were not found in the perivascular location, as was the case for NG2 positive cells. At later stages of angiogenesis, when endothelial cell- populated blood vessels were perfused, cells co-expressing NG2 and F4/80 were down-regulated to 10\% and most NG2 positive cells were reported to have lost CD45 [28].

In summary a large body of evidence describes pericytes with a hematopoietic phenotype, and even more specific, a monocytic origin during blood vessel formation. This is in contrast to pericytes that were isolated from quiescent tissues and demonstrated to be in essence MSCs [41].

In conclusion, it may be hypothesized that at least two independent pericyte populations are involved in angiogenesis (Fig. 1). Both populations can be recruited from the bone marrow. Hematopoietic pericytes are derived from hematopoietic stem cells via the monocytic lineage and contribute to early angiogenic processes such as vessel sprouting.

In contrast, mesenchymal pericytes can be identified as MSCs upon isolation and contribute to later angiogenic processes such as vessel maturation (Fig. 1). This would be in accordance with findings in PDGD-B and PDGFR- $\beta$ knock-out models that showed that lacking (mesenchymal) pericyte recruitment did not affect vessel sprouting [13]. These mesenchymal pericytes were reported to negatively regulate endothelial proliferation and induce vessel maturation and vessel stabilization and remained closely associated with mature vessels $[11,13,32]$. Moreover, hematopoietic pericytes were reported to be present at the onset of angiogenesis and diminished as the vessels matured [28].

\subsection{Monocyte-derived cells in angiogenesis}

Monocyte-derived cells are often referred to as macrophages. As the name 'macrophage' (Greek: big eaters) indicates, monocyte-derived cells are able to phagocytose. However, macrophages are highly heterogeneous cells present in almost all tissues, where they contribute to the host defence, inflamma- 


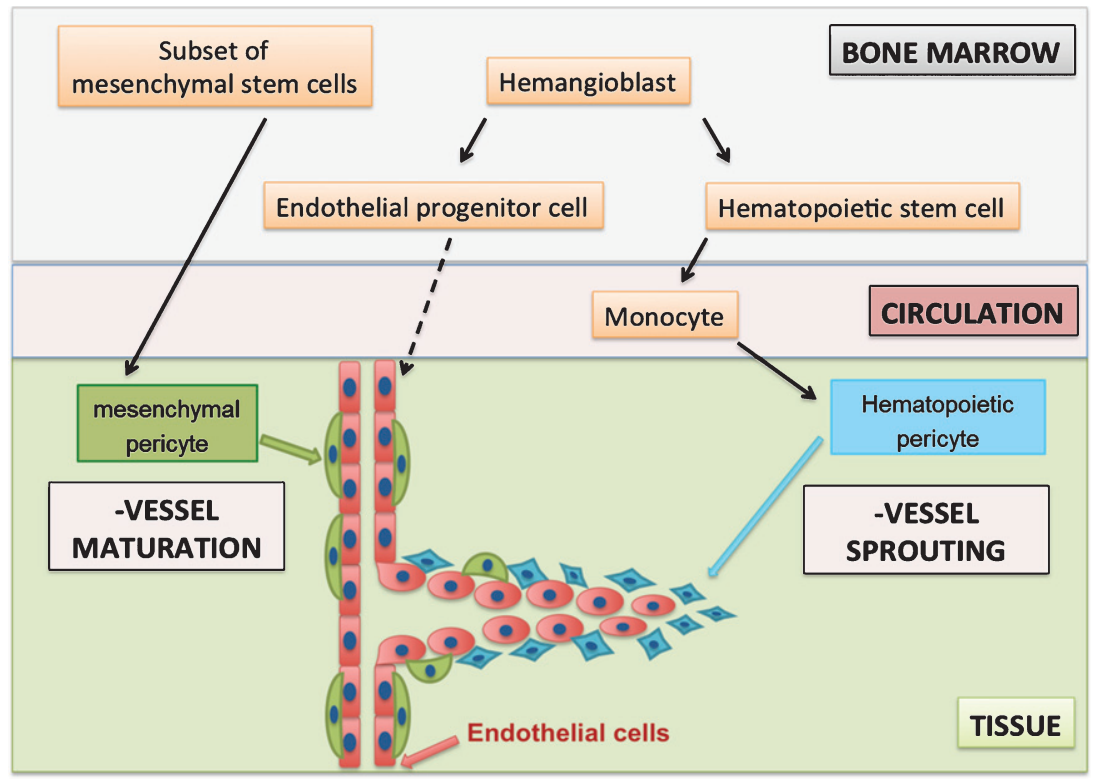

Fig. 1. Hypothesis of two pericyte populations during angiogenesis. Hematopoietic pericytes have a monocytic origin and support early stages of angiogenesis, whereas mesenchymal pericytes are a subset of MSCs, which are present on mature vessels. Mesenchymal pericytes induce vessel maturation and stabilisation at later stages of angiogenesis.

tion and tissue remodelling. They fulfil also highly specialized local functions [64]. Commonly known specialized macrophages are the osteoclasts in the bone, the microglial cells in the central nervous system and the Kupffer cells in the liver as well as histiocytes in connective tissue. Macrophages also exhibit an immense plasticity as they can adapt to their microenvironment by changing their phenotype and function $[4,29]$. In an attempt to classify this plasticity of macrophages a spectrum with two extrema, namely M1 and M2, was suggested with macrophages taking up any phenotype in between those extrema [64]. M1 represent classically activated macrophages, which secrete high levels of pro-inflammatory cytokines, and are thus microbicidal and tumouricidal. M2 macrophages have a decreased ability to present antigens, secrete minimal amount of pro-inflammatory cytokines and even act as immune-modulatory and wound healing promoting cells [64, 65].

\subsubsection{Macrophages in the formation of vasculature during development}

Macrophages have been detected in early stages of embryogenesis [66]. The first macrophages present in the yolk sac of the embryo were of maternal origin. Subsequent macrophages were of embryonic origin. Interestingly, maternal and embryonic macrophages exhibited a M2 phenotype with enriched mRNA of genes associated with wound healing and angiogenesis. [66].

During the vascularisation of the hindbrain, macrophages were observed to migrate from the yolk sac into the embryo proper before the vascularisation was established [67]. Macrophages were often in contact with endothelial tip cells and bridged the space between two tip cells during the formation of the vasculature, thus directing anastomosis formation. Similarly, during postnatal vascularization of the retina, infiltration of macrophages has been observed before the establishment of the vasculature with these macrophages bridging fusing blood vessels [67, 68]. Interestingly, vasculature in knock-out models lacking macrophages appeared to be sparser and to have fewer intersections [67, 68]. An in vitro functional angiogenesis assay utilizing aortic rings demonstrated that in the presence of microglia sprouts appeared earlier and more sprouts developed in the presence of microglia [68]. 


\subsubsection{Macrophages in angiogenesis during adulthood}

During would healing, macrophages with various distinct phenotypes play a crucial role in the acute inflammatory response and tissue remodelling including angiogenesis. When macrophages were depleted from the acute inflammatory phase, angiogenesis was strongly impaired. In contrast when macrophages were depleted from the proliferative phase, haemorrhages appeared in the formed granulation tissue. Macrophages are well known to secrete pro-angiogenic factors, and although it remains to be confirmed which macrophage populations contribute to various angiogenic phases, it is certain that they are indispensable for angiogenesis in adulthood.

In tumours, malignancy is linked to a high density of the vasculature within the tumour, the so-called angiogenic switch [69]. It was reported that the onset of the angiogenic switch was delayed, when infiltration of macrophages into tumours was inhibited [69]; indeed a high tumour-associated macrophage (TAM) presence was associated with increased microvessel density [70], which was associated with a poor prognosis [71]. Various phenotypes of monocytes and macrophages with differing functions in the tumour were identified as TAM [72]. M1 macrophages, which secreted high levels of cytotoxic cytokines, such as TNF or IL-12 within the tumour, supposedly suppressed tumour growth [70, 71]. On the other hand M2 macrophages promoted tumour progression with various pro-angiogenic immune-suppressive cytokines [73]. Furthermore, they secreted various proteases, digesting ECM and thereby promoting endothelial sprouting and metastasis [70, 71]. As macrophages are highly plastic, it is assumed that the tumour cells and/or tumour environment influence monocytes to polarise into a M2 phenotype [71].

Monocyte-derived cells take up distinct phenotypes and associated functions in angiogenesis; hence one of the essential cell types might very well be hematopoietic pericytes.

\section{Sourcing hematopoietic pericytes from peripheral blood}

Very recently Yamazaki and colleagues isolated embryonic myeloid progenitors from murine skin and demonstrated their ability differentiate into pericytes [30].

Two years prior to this we published a study that described the isolation of a cell population from human adult peripheral blood closely resembling hematopoietic pericytes. Peripheral blood mononuclear cells (PBMCs) were cultured as a crude mixture under mixed macromolecular crowding (MMC) [74]. The crowded conditions that are normally found in vivo are emulated in vitro by the addition of polysucrose molecules $[75,76]$. This macromolecular crowding has many downstream effects on protein-protein interaction and assembly, enzyme kinetics and can also act as a signal amplifier [66, 75]. After five days of culture, most adherent cells had a spindle-shape morphology and expressed pan-leukocyte marker CD45 and monocyte marker CD11b, as described for hematopoietic pericytes in vivo. More importantly, independent of the blood sample, these spindle-shaped cells always expressed pericyte markers, PDGFR-B, NG2 and Tie-2. Desmin expression was also observed, but it was variable from blood sample to sample. In addition, they lacked MSCs markers such as CD146, thus were distinguishable from mesenchymal pericytes and expressed many angiogenic markers, such as VEGFR-1 and VEGFR-2. Although these cells had a monocytic origin, they exhibited a stable phenotype, as they could not be polarized towards M1 or M2 macrophages, nor did they exhibit macrophage features such as the ability to phagocytose [74]. Thus, although they were monocyte derived, they did not resemble macrophages.

Spindle-shaped cells were also subjected to various functional assays, and were demonstrated to co-localize with endothelial tubular networks and to dramatically enhance endothelial sprouting in vitro [74]. Thus, we termed this cell type blood-derived angiogenic cells (BDACs). 

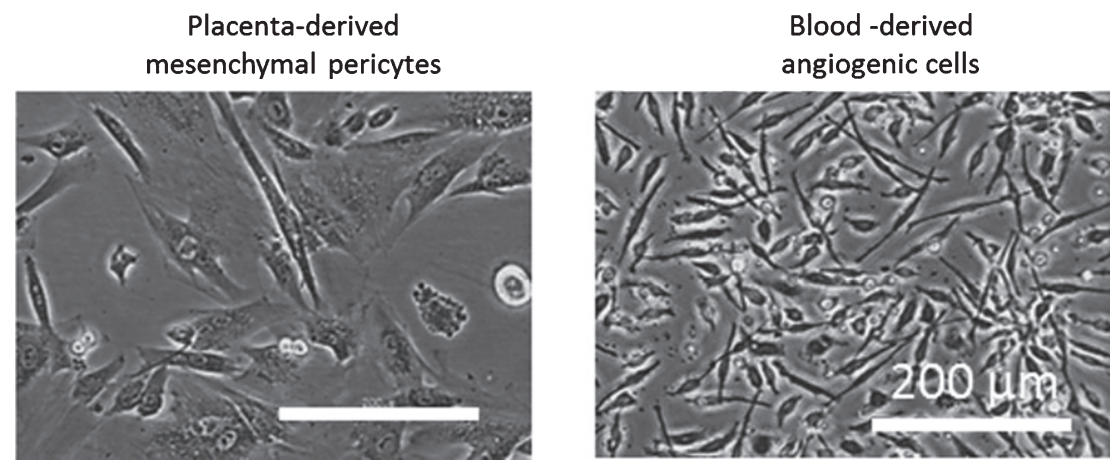

Fig. 2. Phase contrast images of placenta-derived mesenchymal pericytes and blood-derived angiogenic cells, which resemble hematopoietic pericytes.

Their contribution to angiogenesis in vivo was studied in a tumour model. In general, when tumour cells are inoculated subcutaneously, the formed tissue vascularizes through ingrowth of vessels from a border zone. Thus, after one week the formed tumour has strongly vascularized areas, an avascular core and sides of angiogenesis in between these two areas. We found that BDACs were absent from the vascularized areas, but persisted at avascular areas as well as sides of angiogenesis. At sides of angiogenesis they often were detected at a perivascular location. Furthermore, a significantly higher vessel density was observed in the formed tumour in the presence of BDACs [74]. Hence, BDACs not only proved to be pro-angiogenic in vivo, but to resemble hematopoietic pericytes that were only observed in vivo thus far.

\subsection{Role of mesenchymal and hematopoietic pericytes during angiogenesis}

The sourcing of hematopoietic pericytes as BDACs opened up the possibility to study and compare both pericyte populations in vitro. We subjected isolated BDACs (Fig. 2) as well as mesenchymal pericytes (Fig. 2) to a functional angiogenesis assay in vitro (Figs. 3 and 4).

For these assays, endothelial cell spheroids were embedded in a collagen I gel and induced to sprout. Both cell populations were added either into the spheroid or as a single cell suspension into the collagen I gel (Figs. 3 and 4). As a result, we found that the presence of mesenchymal pericytes around spheroids inhibited endothelial sprouting in a dose dependent manner (Fig. 3a). When mesenchymal pericytes were added directly in the spheroids, fewer sprouts were observed, although the sprouts exhibited a smoother and more continuous morphology (Fig. 3b) [58].

In contrast, BDACs enhanced endothelial sprouting in a dose dependent manner when added into the collagen I gel (Fig. 2d) [74]. Upon direct addition of BDACs into the spheroids, it was observed that spheroids often disintegrated into several spheroids of smaller size, indicating that BDACs also induced endothelial cell mobilization (Fig. 2d) [74].

Interestingly, both cells populations co-localized with forming sprouts, as expected from pericytes. Thus, we could confirm in vitro that BDACs, while resembling hematopoietic pericytes, supported early angiogenic processes such as endothelial mobilization and sprouting, whereas mesenchymal pericytes did not support the initial sprouting process, but stabilized already formed sprouts.

\subsection{Other non-conventional monocyte-derived cells}

Other non-conventional monocyte-derived cells generated in vitro that shared features with BDACs do exist. Such cell types are known as fibrocytes, endothelial progenitor cells or endothelial like cells. They all share a spindle-shaped cell morphology and several common markers. 
a)
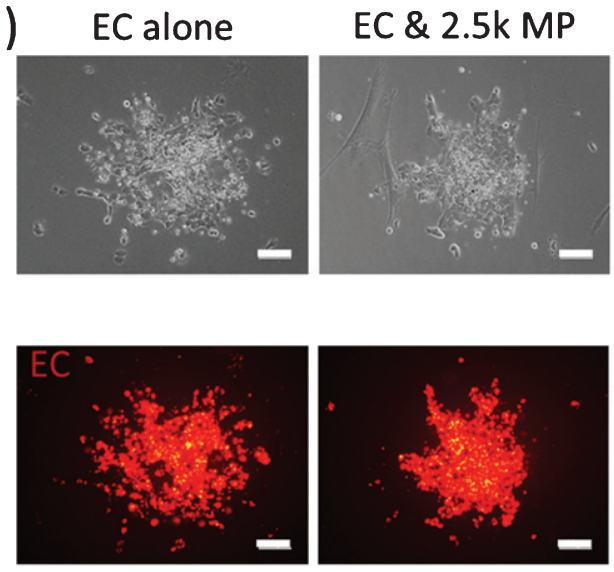

b)
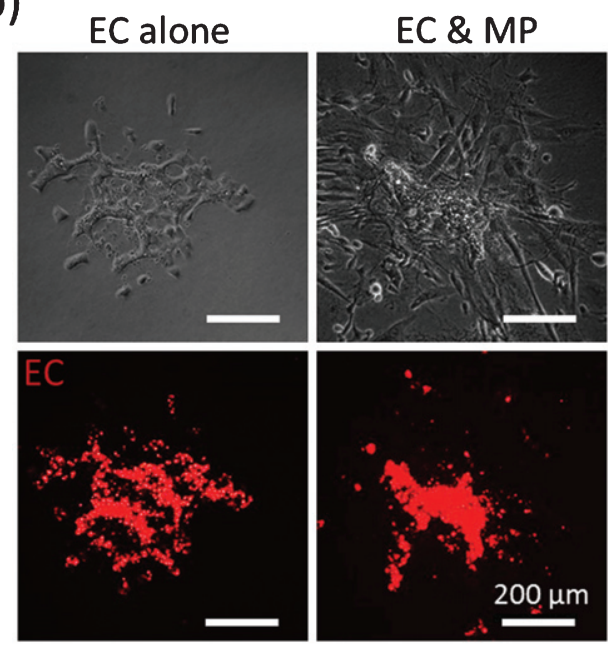
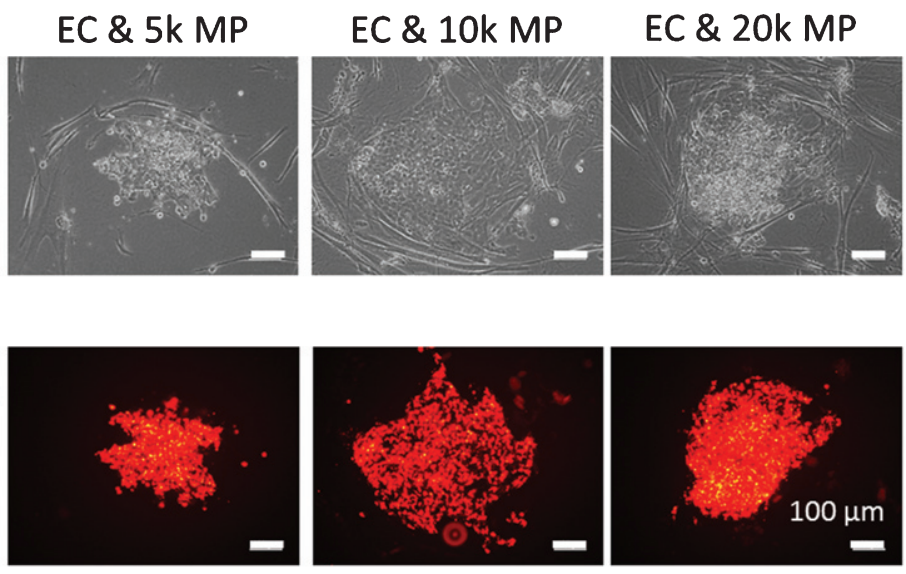

\section{Normalized cumulative sprout length per spheroid}

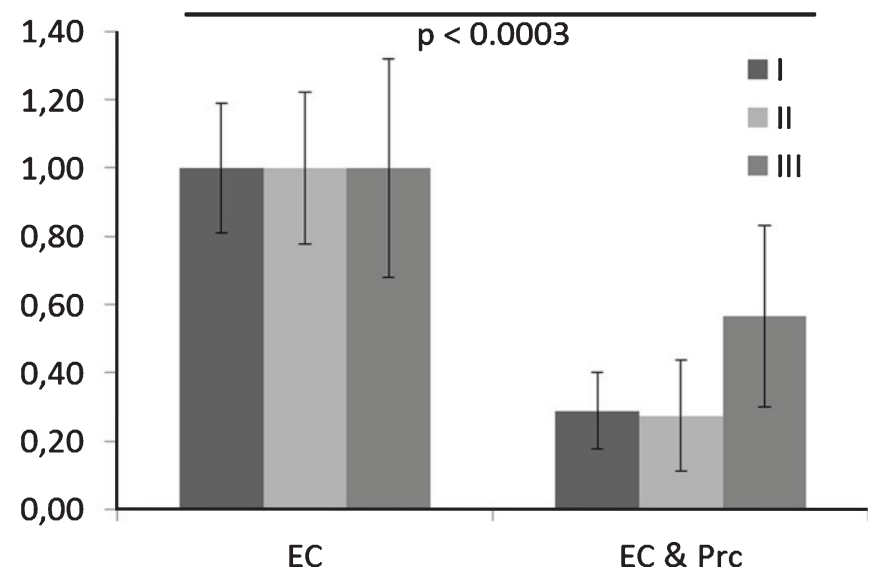

Fig. 3. Spheroid sprouting assay: Endothelial cells (EC, red staining) were embedded in a collagen I gel and allowed to sprout. a) Mesenchymal pericytes (MP) were added as a single cell suspension directly into the gel. MP concentrations are displayed as cell number per ml. b) Mixed spheroids containing EC (red staining) and MP were embedded in a collagen I gel and allowed to sprout.

\subsubsection{Fibrocytes}

Fibrocytes were first characterised in vivo by Bucala and colleagues in 1994 [77]. In 2001, they were able to isolate this cell type in vitro from peripheral blood [78]. Fibrocytes were identified as monocyte-derived, and express the extracellular matrix protein collagen I, although only intracellular [78]. Fibrocytes were potent antigen-presenting cells expressing the MHC II complex, and could efficiently stimulate a T-cell response [79]. Interestingly cytokines that are known to induce M2 polarisation in macrophages promoted the appearance of fibrocytes in culture, whereas cytokines that are known to induce an M1 phenotype, inhibited fibrocyte differentiation [80].

Fibrocytes were studied for their pro-angiogenic attributes, and were found to secrete many proangiogenic factors including MMP9, VEGF, bFGF, PDGF, IL-8, GM-CSF and angiogenin [81]. Interestingly, a similar secretion panel was described for TAM [70, 71]. In vivo, fibrocytes enhanced vascularization of a matrigel plug [81] and homed into areas of wound healing, with some fibrocytes localised around newly formed blood vessels [78]. 
a)

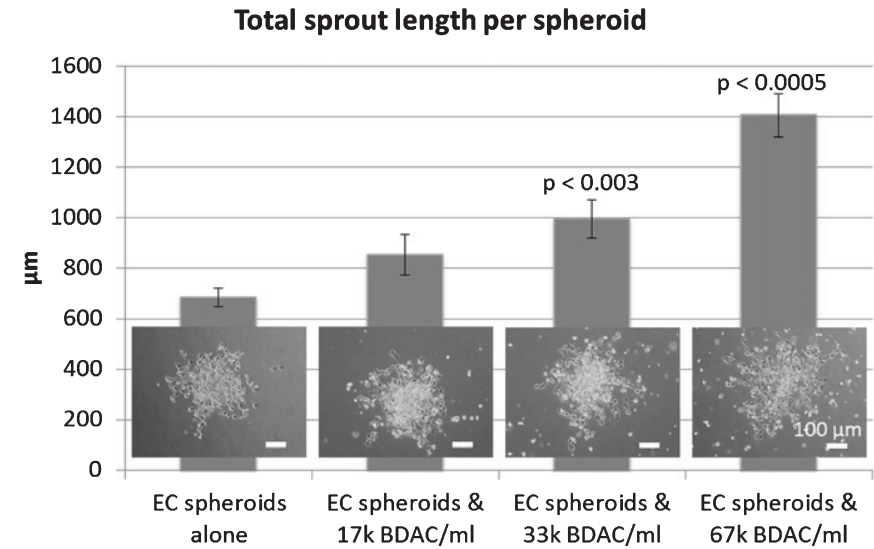

b)

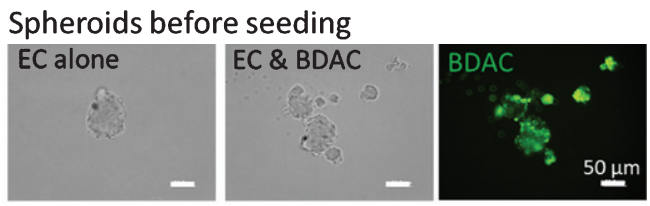

Mixed spheroids (EC \& BDAC) in collagen I gel

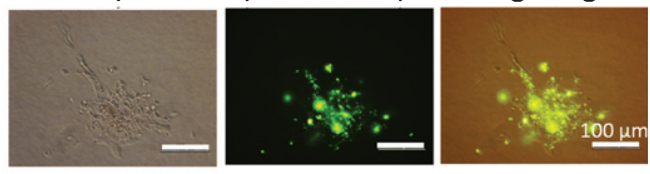

Fig. 4. Spheroid sprouting assay: Endothelial cells were embedded in a collagen I gel and allowed to sprout. a) Mixed spheroids containing EC and BDAC (green staining) were embedded in a collagen I gel and allowed to sprout. b) BDACs were added as a single cell suspension directly into the gel.

\subsubsection{Endothelial-like cells}

Endothelial cells form the luminal wall of blood vessels and represent a dynamic interface between circulating blood and surrounding tissue. Generally it is believed that hematopoietic stem cell (HSC) and endothelial cells share a common progenitor, namely the hemangioblast, located in the bone marrow $[82,83]$. The hemangioblast gives rise to an early endothelial progenitor cell (EPC), which shares markers with HSCs, CD133 and CD34. Upon entry into the circulation, the early EPC matures as indicated by the expression of CD144 (VE-Cadherin) and vWF, while losing CD133 at the same time. Once this cell type matures it is often referred to as late endothelial progenitor cells (EPC) [82, 84]. It is important to note that the hemangioblast does not express any leukocyte (CD45) or monocyte (CD14) markers during any stage of differentiation.

Similar to BDACs or fibrocytes, endothelial progenitor cells can be isolated from peripheral blood whilst culturing whole PBMCs [85]. After two weeks of culture, small colonies with cobble-stone morphology grew out and were identified as late EPC. Interestingly, before late EPC colonies emerged, spindle-shaped cells appeared which were referred to as endothelial like cells (ELC) or early EPCs. In contrast to proper endothelial cells such as late EPC, they did not express all endothelial markers such as eNOS [86] or CD146 [87], but exhibited the monocyte marker CD14, and did not form tubes on matrigel on their own $[79,88]$. Early EPC were derived from monocytes and secreted MMP9 [89]. They were shown to express many endothelial markers such as CD34, CD31, VEGFR-1 and -2, VE-Cadherin, CD105 and vWF [89], and to secrete angiogenic factors like VEGF and IL-8 [88]. Interestingly, early ELC and fibrocytes seem to be very similar in terms of morphology and marker expression and are both derived from monocytes [90]. BDACs were clearly distinguishable from ELC and fibrocytes, as they lacked ELC markers VE-Cadherin and VWF and fibrocyte marker collagen I and expressed various pericyte markers. However, BDACs secreted common pro-angiogenic factors such as MMP-9, GM-CSF, VEGF, and IL-8 HB-EGF and MCP-1 that partially overlap with the secretome of fibrocytes, ELC and TAM pointing to a partial overlap in the mode of action. Since monocytes are very plastic and can give rise to cells with varying phenotypes between M1 and M2 [64], it is likely that fibrocytes, ELC and BDACs are related. 


\subsection{Pericytes in therapeutic angiogenesis}

The enhancement of revascularization by means of supporting angiogenesis is essential for the treatment of ischemic diseases such as stroke, myocardial infarction or critical limb ischemia.

Main strategies for therapeutic angiogenesis include delivery of genes and growth factors, and cell-based therapies [91]. Growth factor administration and gene therapy employ only selected factors as therapeutic agents. However, these often show adverse side-effects due to uncontrolled non-physiological doses. Additionally in clinical trials, growth factor delivery failed to demonstrate successful improvements upon administration, limited by rapid diffusion and poor stability in vivo $[92,93]$. Although cell-based therapy offers a more holistic approach, where a range of paracrine factors is secreted locally in response to host tissue signals, the clinical success has nevertheless been limited [94]. These cellular therapies invariably involve the application of mainly crude cellular populations derived from bone marrow mononucleated cells or further purified sub-populations such as EPCs or MSCs. Results of clinical trials for cell-based therapies so far have only shown a benefit for patients suffering from peripheral artery disease [95-97]. However, results from similar cell-based therapies of cardiac ischemic diseases have been unconvincing [98, 99].

In pre-clinical studies, the utilization of mesenchymal pericytes showed promising results. Upon delivery into a myocardial infarct, mesenchymal pericytes derived from skeletal muscle reduced fibrosis and improved cardiac function. They acted as immunomodulatory and proangiogenic, although only a fraction of transplanted cells took up a perivascular location [100]. In conjunction, pericytes derived from the saphenous vein supported cardiac function and modulated cardiac wall thinning, while also enhancing angiogenesis in the peri-infarct and infarct area by reducing endothelial cell apoptosis and supporting endothelial cell proliferation [101].

When BDACs were used as a cell-based treatment of critical limb ischemia in a pre-clinical model, they significantly accelerated revascularization resulting in a functional rescue of the affected limb. In contrast control animals suffered from extensive foot necrosis. Ex-vivo analysis proved that BDAC reduced inflammation, fibrosis and adipose replacement in the affected muscle tissues. Since BDACs can be sourced from peripheral blood, which represents an easy accessible and renewable cell source, in clinically relevant numbers within a short period of time (5 days), they represent an attractive cell type for therapeutic angiogenesis [74].

\section{Conclusion}

Although pericytes still represent an elusive cell-type, the existence of several subpopulations of pericytes is well accepted. The participation of at least two pericyte populations during angiogenesis, one mesenchymal and one of hematopoieitic origin, enables a clarification of the discrepancy currently present in literature. We have been also able to provide strong evidence that hematopoietic pericytes promote angiogenic processes such as endothelial sprouting, whereas mesenchymal pericytes induce vessel maturation.

During early stages of angiogenesis, such as endothelial sprouting, the basement membrane is discontinuous and endothelial cells are motile. Thus, it remains difficult to identify pericytes by their close proximity to endothelial cells within the basement membrane during angiogenesis. Since no pericyte specific markers exist, a new pericyte definition was proposed involving the expression of markers such as PDGFR- 3 and/or NG2 in combination with their perivascular location. This broader definition would now encompass a larger set of cell populations including hematopoietic pericytes. 
Nonetheless, independent of their nomenclature, the perivascular cells mentioned herein are indispensable from angiogenesis. The potential of MSC-based cell populations for therapeutic angiogenesis has been well recognized and extensively studied. The identification of mesenchymal pericytes as a subpopulation of MSCs has opened new avenues to employ cells that will specifically target angiogenesis.

However as elaborated in this review, mesenchymal pericytes are involved in later stages of angiogenesis such as vessel maturation, and do not extensively contribute to endothelial sprouting. As the sprouting of new blood vessels is crucial when inducing angiogenesis in diseased tissues, hematopoietic pericytes might represent a valid addition or alternative to MSC-based treatments of ischemic diseases.

\section{Acknowledgments}

AB would like to acknowledge the Institute for Tissue Engineering and Regenerative Medicine at at the Chinese University of Hong Kong for the received funding. Some of the initial ideas leading to this manuscript have been developed at the National University of Singapore and are mentioned in the doctoral dissertation of $\mathrm{AB}$.

\section{Dedication}

Anna Blocki would like to dedicate this review to the 70th anniversary of Prof. Friedrich Jung and thank him for the many scientific fruitful discussions they shared.

\section{References}

[1] Sims DE. The pericyte-a review. Tissue Cell. 1986;18(2):153-74.

[2] Wu Q, Jing Y, Yuan X, Li B, Wang B, Liu M, et al. The distinct abilities of tube-formation and migration between brain and spinal cord microvascular pericytes in rats. Clin Hemorheol Microcirc. 2015;60(2):231-40.

[3] Rouget C. Memoire sur le developpement, la strucutures et les proprieties des capillaires sanguins et lymphatiques. Archs Physiol Norm Pathol. 1873;5603-33.

[4] Prazeres DMPH, Sena IFG, Borges IDT, de Azevedo PO, Andreotti JP, de Paiva AE, et al. Pericytes are heterogeneous in their origin within the same tissue. Dev Biol. 2017;427(1):6-11.

[5] Song S, Ewald AJ, Stallcup W, Werb Z, Bergers G. PDGFRbeta+ perivascular progenitor cells in tumours regulate pericyte differentiation and vascular survival. Biol. 2005;7(9):870-9.

[6] Rajantie I, Ilmonen M, Alminaite A, Ozerdem U, Alitalo K, Salven P. Adult bone marrow-derived cells recruited during angiogenesis comprise precursors for periendothelial vascular mural cells. Blood. 2004;104(7):2084-6.

[7] Kidd S, Spaeth E, Watson K, Burks J, Lu H, Klopp A, et al., Origins of the tumor microenvironment: Quantitative assessment of adipose-derived and bone marrow-derived stroma. PloS One. 2012;7(2):e30563.

[8] Kokovay E, Li L, Cunningham LA. Angiogenic recruitment of pericytes from bone marrow after stroke. Journal of Cerebral Blood Flow \&\#38; Metabolism. 2005;26(4):545-55.

[9] Leveen P, Pekny M, Gebre-Medhin S, Swolin B, Larsson E, Betsholtz C. Mice deficient for PDGF B show renal, cardiovascular, and hematological abnormalities. Genes Dev. 1994;8(16):1875-87.

[10] Soriano P. Abnormal kidney development and hematological disorders in PDGF beta-receptor mutant mice. Genes Dev. 1994;8(16):1888-96.

[11] Gerhardt H, Betsholtz C. Endothelial-pericyte interactions in angiogenesis. Cell Tissue Res. 2003;314(1):15-23.

[12] Gaengel K, Genove G, Armulik A, Betsholtz C. Endothelial-mural cell signaling in vascular development and angiogenesis. (1). Arterioscler Thromb Vasc Biol. 2009;29(5):630-8.

[13] Hellström M, Gerhardt H, Kalén M, Li X, Eriksson U, Wolburg H, et al. Lack of pericytes leads to endothelial hyperplasia and abnormal vascular morphogenesis. J Cell Biol. 2001;153(3):543-53. 
[14] Orlidge A, D'Amore PA. Inhibition of capillary endothelial cell growth by pericytes and smooth muscle cells. J Cell Biol. 1987;105(3):1455-62.

[15] Terlizzi V, Kolibabka M, Burgess JK, Hammes HP, Harmsen MC. The pericytic phenotype of adipose tissue-derived stromal cells is promoted by NOTCH2. Stem Cells. 2017. DOI: 10.1002/stem.2726

[16] Teichert M, Milde L, Holm A, Stanicek L, Gengenbacher N, Savant S, et al. Pericyte-expressed Tie2 controls angiogenesis and vessel maturation. Nat Commun. 2017;8161038. DOI: 10.1038/ncomms 16106

[17] Abramsson A. Analysis of mural cell recruitment to tumor vessels. Circulation. 2002;105(1):112-7.

[18] Rajkumar VS, Shiwen X, Bostrom M, Leoni P, Muddle J, Ivarsson M, et al. Platelet-derived growth factor- $\beta$ receptor activation is essential for fibroblast and pericyte recruitment during cutaneous wound healing. Am $\mathrm{J}$ Pathol. 2006;169(6):2254-65.

[19] Ozerdem U, Monosov E, Stallcup WB. NG2 proteoglycan expression by pericytes in pathological microvasculature. Microvasc Res. 2002;63(1):129-34.

[20] Ponce AM, Price RJ. Angiogenic stimulus determines the positioning of pericytes within capillary sprouts in vivo. Microvasc Res. 2003;65(1):45-8.

[21] Stapor PC, Sweat RS, Dashti DC, Betancourt AM, Murfee WL. Pericyte dynamics during angiogenesis: New insights from new identities. J Vasc Res. 2014;51(3):163-74.

[22] Bergers G, Song S. The role of pericytes in blood-vessel formation and maintenance. Neuro Oncol. 2005;7(4):452-64.

[23] Birbrair A, Zhang T, Wang ZM, Messi ML, Mintz A, Delbono O. Pericytes at the intersection between tissue regeneration and pathology. Clin Sci (Lond). 2015;128(2):81-93.

[24] Crisan M, Chen CW, Corselli M, Andriolo G, Lazzari L, Péault B. Perivascular multipotent progenitor cells in human organs. Ann N Y Acad Sci. 2009;1176118-23.

[25] Peault B. Are mural cells guardians of stemness? From pluri- to multipotency via vascular pericytes. Circulation. 2012;125(1):12-3.

[26] Ozerdem U. Contribution of bone marrow-derived pericyte precursor cells to corneal vasculogenesis. Invest Ophthalmol Vis Sci. 2005;46(10):3502-6.

[27] Lamagna C, Bergers G. The bone marrow constitutes a reservoir of pericyte progenitors. J Leukoc Biol. 2006;80(4):677-81.

[28] Tigges U, Hyer EG, Scharf J, Stallcup WB. FGF2-dependent neovascularization of subcutaneous Matrigel plugs is initiated by bone marrow-derived pericytes and macrophages. Development. 2008;135(3):523-32.

[29] Prazeres PHDM, Almeida VM, Lousado L, Andreotti JP, Paiva AE, Santos GSP, et al. Macrophages generate pericytes in the developing brain. Cell Mol Neurobiol. 2017. DOI: 10.1007/s10571-017-0549-2

[30] Yamazaki T, Nalbandian A, Uchida Y, Li W, Arnold TD, Kubota Y, et al. Tissue myeloid progenitors differentiate into pericytes through TGF- $\beta$ signaling in developing skin vasculature. Cell Rep. 2017;18(12):2991-3004.

[31] Dominici M, Le Blanc K, Mueller I, Slaper-Cortenbach I, Marini F, Krause D, et al. Minimal criteria for defining multipotent mesenchymal stromal cells. The International Society for Cellular Therapy Position Statement. Cytotherapy. 2006;8(4):315-7.

[32] Lindahl P, Johansson BR, Levéen P, Betsholtz C. Pericyte loss and microaneurysm formation in PDGF-B-deficient mice. Science. 1997;277(5323):242-5.

[33] Hellström M, Kalén M, Lindahl P, Abramsson A, Betsholtz C. Role of PDGF-B and PDGFR-beta in recruitment of vascular smooth muscle cells and pericytes during embryonic blood vessel formation in the mouse. Development. 1999;126(14):3047-55.

[34] Rajkumar VS, Shiwen X, Bostrom M, Leoni P, Muddle J, Ivarsson M, et al. Platelet-derived growth factor- $\beta$ receptor activation is essential for fibroblast and pericyte recruitment during cutaneous wound healing (1). Am J Pathol. 2006;169(6):2254-65.

[35] Armulik A, Genové G, Betsholtz C. Pericytes: Developmental, physiological, and pathological perspectives, problems, and promises. Dev Cell. 2011;21(2):193-215.

[36] Chen J, Luo Y, Huang H, Wu S, Feng J, Zhang J, et al. CD146 is essential for PDGFR $\beta$-induced pericyte recruitment. Protein Cell. 2017. DOI: 10.1007/s13238-017-0484-5

[37] Canfield AE, Sutton AB, Hoyland JA, Schor AM. Association of thrombospondin-1 with osteogenic differentiation of retinal pericytes in vitro. J Cell Sci. 1996;109(Pt 2)343-53.

[38] Brighton CT, Lorich DG, Kupcha R, Reilly TM, Jones AR, Woodbury RA. The pericyte as a possible osteoblast progenitor cell. Clin Orthop Relat Res. 1992;(275):287-99.

[39] Hirschi KK, D’Amore PA. Pericytes in the microvasculature. Cardiovasc Res. 1996;32(4):687-98.

[40] Farrington-Rock C. Chondrogenic and adipogenic potential of microvascular pericytes. Circulation. 2004;110(15):2226-32. 
[41] Crisan M, Yap S, Casteilla L, Chen CW, Corselli M, Park TS, et al. A perivascular origin for mesenchymal stem cells in multiple human organs. Cell Stem Cell. 2008;3(3):301-13.

[42] Chen WC, Baily JE, Corselli M, Díaz ME, Sun B, Xiang G, et al. Human myocardial pericytes: Multipotent mesodermal precursors exhibiting cardiac specificity. Stem Cells. 2015;33(2):557-73.

[43] Diaz-Flores L, Gutierrez R, Lopez-Alonso A, Gonzalez R, Varela H. Pericytes as a supplementary source of osteoblasts in periosteal osteogenesis. Clin Orthop Relat Res. 1992;(275):280-6.

[44] Feng J, Mantesso A, De Bari C, Nishiyama A, Sharpe PT. Dual origin of mesenchymal stem cells contributing to organ growth and repair. Proc Natl Acad Sci U S A. 2011;108(16):6503-8.

[45] Tang W, Zeve D, Suh JM, Bosnakovski D, Kyba M, Hammer RE, et al. White fat progenitor cells reside in the adipose vasculature. Science. 2008;322(5901):583-6.

[46] Dellavalle A, Sampaolesi M, Tonlorenzi R, Tagliafico E, Sacchetti B, Perani L, et al. Pericytes of human skeletal muscle are myogenic precursors distinct from satellite cells. Nat Cell Biol. 2007;9(3):255-67.

[47] Park TS, Gavina M, Chen CW, Sun B, Teng PN, Huard J, et al. Placental perivascular cells for human muscle regeneration. Stem Cells Dev. 2011;20(3):451-63.

[48] Zebardast N, Lickorish D, Davies JE. Human umbilical cord perivascular cells (HUCPVC): A mesenchymal cell source for dermal wound healing. Organogenesis. 2010;6(4):197-203.

[49] Schrimpf C, Duffield JS. Mechanisms of fibrosis: The role of the pericyte. Curr Opin Nephrol Hypertens. 2011;20(3):297-305.

[50] Guimarães-Camboa N, Cattaneo P, Sun Y, Moore-Morris T, Gu Y, Dalton ND, et al. Pericytes of multiple organs do not behave as mesenchymal stem cells in vivo. Cell Stem Cell. 2017;20(3):345-59.e5.

[51] Cano E, Gebala V, Gerhardt H. Pericytes or mesenchymal stem cells: Is that the question? Cell Stem Cell. 2017;20(3):296-7.

[52] Birbrair A, Zhang T, Files DC, Mannava S, Smith T, Wang ZM, et al. Type-1 pericytes accumulate after tissue injury and produce collagen in an organ-dependent manner. Am J Physiol Cell Physiol. 2014;307(1):C25-38.

[53] Caplan AI. All MSCs are pericytes? (1). Cell Stem Cell. 2008;3(3):229-30.

[54] da Silva Meirelles L. Mesenchymal stem cells reside in virtually all post-natal organs and tissues. J Cell Sci. 2006;119(11):2204-13.

[55] Horwitz E, Le Blanc K, Dominici M, Mueller I, Slaper-Cortenbach I, Marini F, et al. Clarification of the nomenclature for MSC: The international society for cellular therapy position statement. Cytotherapy. 2005;7(5):393-5.

[56] Corselli M, Chen CW, Crisan M, Lazzari L, Peault B. Perivascular ancestors of adult multipotent stem cells. (1). Arterioscler Thromb Vasc Biol. 2010;30(6):1104-9.

[57] Corselli M, Chen CW, Sun B, Yap S, Rubin JP, Péault B. The tunica adventitia of human arteries and veins as a source of mesenchymal stem cells. Stem Cells Dev. 2012;21(8):1299-308.

[58] Blocki A, Wang Y, Koch M, Peh P, Beyer S, Law P, et al. Not all MSCs can act as pericytes: Functional in vitro assays to distinguish pericytes from other mesenchymal stem cells in angiogenesis. Stem Cells Dev. 2013;22(17):2347-55.

[59] Birbrair A, Zhang T, Wang ZM, Messi ML, Enikolopov GN, Mintz A, et al. Role of pericytes in skeletal muscle regeneration and fat accumulation. Stem Cells Dev. 2013;2(16):2298-314.

[60] Miyamoto T, Sasaguri Y, Sasaguri T, Azakami S, Yasukawa H, Kato S, et al. Expression of stem cell factor in human aortic endothelial and smooth muscle cells. Atherosclerosis. 1997;129(2):207-13.

[61] Sata M, Saiura A, Kunisato A, Tojo A, Okada S, Tokuhisa T, et al. Hematopoietic stem cells differentiate into vascular cells that participate in the pathogenesis of atherosclerosis. Nat Med. 2002;8(4):403-9.

[62] Yamada Y, Takakura N. Physiological pathway of differentiation of hematopoietic stem cell population into mural cells. J Exp Med. 2006;204(4):1055-65.

[63] Lamagna C, Bergers G. The bone marrow constitutes a reservoir of pericyte progenitors. (2). J Leukoc Biol. 2006;80(4):677-81.

[64] Mosser DM, Edwards JP. Exploring the full spectrum of macrophage activation. Nat Rev Immunol. 2008;8(12):958-69.

[65] Mantovani A. Macrophage diversity and polarization: In vivo veritas. Blood. 2006;108(2):408-9.

[66] Nucera S, Biziato D, De Palma M. The interplay between macrophages and angiogenesis in development, tissue injury and regeneration. Int J Dev Biol. 2011;55(4-5):495-503.

[67] Fantin A, Vieira JM, Gestri G, Denti L, Schwarz Q, Prykhozhij S, et al. Tissue macrophages act as cellular chaperones for vascular anastomosis downstream of VEGF-mediated endothelial tip cell induction. Blood. 2010;116(5):829-40.

[68] Rymo SF, Gerhardt H, Wolfhagen Sand F, Lang R, Uv A, Betsholtz C, et al. A two-way communication between microglial cells and angiogenic sprouts regulates angiogenesis in aortic ring cultures. PloS One. 2011;6(1):e15846.

[69] Lin EY, Li JF, Gnatovskiy L, Deng Y, Zhu L, Grzesik DA, et al. Macrophages regulate the angiogenic switch in a mouse model of breast cancer. Cancer Res. 2006;66(23):11238-46. 
[70] Murdoch C, Muthana M, Coffelt SB, Lewis CE. The role of myeloid cells in the promotion of tumour angiogenesis. Nat Rev Cancer. 2008;8(8):618-31.

[71] Sica A, Larghi P, Mancino A, Rubino L, Porta C, Totaro MG, et al. Macrophage polarization in tumour progression. Semin Cancer Biol 2008;18(5):349-55.

[72] Laurent J, Touvrey C, Botta F, Kuonen F, Ruegg C. Emerging paradigms and questions on pro-angiogenic bone marrow-derived myelomonocytic cells. Int J Dev Biol. 2011;55(4-5):527-34.

[73] De Palma M, Venneri MA, Galli R, Sergi LS, Politi LS, Sampaolesi M, et al. Tie2 identifies a hematopoietic lineage of proangiogenic monocytes required for tumor vessel formation and a mesenchymal population of pericyte progenitors. Cancer Cell. 2005;8(3):211-26.

[74] Blocki A, Wang Y, Koch M, Goralczyk A, Beyer S, Agarwal N, et al. Sourcing of an alternative pericyte-like cell type from peripheral blood in clinically relevant numbers for therapeutic angiogenic applications. Mol Ther. 2015;23(3):510-22.

[75] Dewavrin JY, Abdurrahiem M, Blocki A, Musib M, Piazza F, Raghunath M. Synergistic rate boosting of collagen fibrillogenesis in heterogeneous mixtures of crowding agents. J Phys Chem B. 2015;119(12):4350-8.

[76] Chen C, Loe F, Blocki A, Peng Y, Raghunath M. Applying macromolecular crowding to enhance extracellular matrix deposition and its remodeling in vitro for tissue engineering and cell-based therapies. Adv Drug Deliv Rev. 2011;63(45):277-90.

[77] Bucala R, Spiegel LA, Chesney J, Hogan M, Cerami A. Circulating fibrocytes define a new leukocyte subpopulation that mediates tissue repair. Mol Med. 1994;1(1):71-81.

[78] Abe R, Donnelly SC, Peng T, Bucala R, Metz CN. Peripheral blood fibrocytes: Differentiation pathway and migration to wound sites. Immunology. 2001;166(12):7556-62.

[79] Chesney J, Bacher M, Bender A, Bucala R. The peripheral blood fibrocyte is a potent antigen-presenting cell capable of priming naive T cells in situ. Proc Natl Acad Sci U S A. 1997;94(12):6307-12.

[80] Shao DD, Suresh R, Vakil V, Gomer RH, Pilling D. Pivotal Advance: Th-1 cytokines inhibit, and Th-2 cytokines promote fibrocyte differentiation. J Leukoc Biol. 2008;83(6):1323-33.

[81] Hartlapp I, Abe R, Saeed RW, Peng T, Voelter W, Bucala R, et al. Fibrocytes induce an angiogenic phenotype in cultured endothelial cells and promote angiogenesis in vivo. FASEB Journal: Official Publication of the Federation of American Societies for Experimental Biology. 2001;15(12):2215-24.

[82] Hristov M. Endothelial progenitor cells: Mobilization, differentiation, and homing. Arterioscler Thromb Vasc Biol. 2003;23(7):1185-9.

[83] Lin Y, Weisdorf DJ, Solovey A, Hebbel RP. Origins of circulating endothelial cells and endothelial outgrowth from blood. J Clin Invest. 2000;105(1):71-7.

[84] Asahara T, Murohara T, Sullivan A, Silver M, Zee RVD, Li T, et al. Isolation of putative progenitor endothelial cells for angiogenesis. Science. 1997;275(5302):964-7.

[85] Ingram DA, Mead LE, Tanaka H, Meade V, Fenoglio A, Mortell K, et al. Identification of a novel hierarchy of endothelial progenitor cells using human peripheral and umbilical cord blood. Blood. 2004;104(9):2752-60.

[86] Gulati R. Diverse origin and function of cells with endothelial phenotype obtained from adult human blood. Circ Res. 2003;93(11):1023-5.

[87] Fuchs S, Hermanns MI, Kirkpatrick CJ. Retention of a differentiated endothelial phenotype by outgrowth endothelial cells isolated from human peripheral blood and expanded in long-term cultures. Cell Tissue Res. 2006;326(1):79-92.

[88] Hur J. Characterization of two types of endothelial progenitor cells and their different contributions to neovasculogenesis. Arterioscler Thromb Vasc Biol. 2004;24(2):288-93.

[89] Yoon CH. Synergistic neovascularization by mixed transplantation of early endothelial progenitor cells and late outgrowth endothelial cells: The role of angiogenic cytokines and matrix metalloproteinases. Circulation. 2005;112(11):1618-27.

[90] Kuwana M, Okazaki Y, Kodama H, Satoh T, Kawakami Y, Ikeda Y. Endothelial differentiation potential of human monocyte-derived multipotential cells. Stem Cells. 2006;24(12):2733-43.

[91] Russo V, Young S, Hamilton A, Amsden BG, Flynn LE. Mesenchymal stem cell delivery strategies to promote cardiac regeneration following ischemic injury. Biomaterials. 2014;35(13):3956-74.

[92] Segers VFM, Lee RT. Protein therapeutics for cardiac regeneration after myocardial infarction. Journal of Cardiovascular Translational Research. 2010;3(5):469-77.

[93] Preda MB, Valen G. Evaluation of gene and cell-based therapies for cardiac regeneration. Curr Stem Cell Res Ther. 2013;8(4):304-12.

[94] Bronckaers A, Hilkens P, Martens W, Gervois P, Ratajczak J, Struys T, et al. Mesenchymal stem/stromal cells as a pharmacological and therapeutic approach to accelerate angiogenesis. Pharmacol Ther. 2014;143(2):181-96. 
[95] Tongers J, Roncalli JG, Losordo DW. Therapeutic angiogenesis for critical limb ischemia: Microvascular therapies coming of age. Circulation. 2008;118(1):9-16.

[96] Gupta NK, Armstrong EJ, Parikh SA. The current state of stem cell therapy for peripheral artery disease. Curr Cardiol Rep. 2014;16(2):447.

[97] Gupta R, Losordo DW. Cell therapy for critical limb ischemia: Moving forward one step at a time. Circulation Cardiovascular Interventions. 2011;4(1):2-5.

[98] Ni NC, Li RK, Weisel RD. The promise and challenges of cardiac stem cell therapy. Semin Thorac Cardiovasc Surg. 2014;26(1):44-52.

[99] Richardson JD, Nelson AJ, Zannettino ACW, Gronthos S, Worthley SG, Psaltis PJ. Optimization of the cardiovascular therapeutic properties of mesenchymal stromal/stem cells-taking the next step. Stem Cell Rev. 2013;9(3):281-302.

[100] Chen CW, Okada M, Proto JD, Gao X, Sekiya N, Beckman SA, et al. Human pericytes for ischemic heart repair. Stem Cells. 2013;31(2):305-16.

[101] Katare R, Riu F, Mitchell K, Gubernator M, Campagnolo P, Cui Y, et al. Transplantation of human pericyte progenitor cells improves the repair of infarcted heart through activation of an angiogenic program involving micro-RNA-132. Circ Res. 2011;109(8):894-906. 